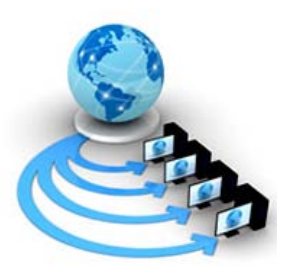

Volume 8, No. 9, November-December 2017

International Journal of Advanced Research in Computer Science

RESEARCH PAPER

Available Online at www.ijarcs.info

\title{
OVERVIEW OF COGNITIVE RADIO NETWORKS
}

\author{
Shelly Berwal \\ M.Tech. Student, Department of Electronics and \\ Communication Engineering, University Institute of \\ Engineering and Technology, Maharshi Dayanand \\ University, Rohtak, Haryana, India
}

\author{
Vikas Sindhu \\ Assistant Professor, Department of Electronics and \\ Communication Engineering, University Institute of \\ Engineering and Technology, Maharshi Dayanand \\ University, Rohtak, Haryana, India
}

\begin{abstract}
In today's era, there is rapid increase in use of wireless technologies. The main backbone of wireless technology is radio spectrum. As radio spectrum is allocated to the fixed users due to which it becomes sometimes underutilized and sometimes scarce. To efficiently use the spectrum bands, Cognitive radio (CR) technology was developed which adapts to the real-time changes in the parameters without causing any interference to other networks. Cognitive radio network helps to allocate the bands to the unlicensed users too when they are not used by the fixed (licensed) users. This paper gives an overview of the CR networks.
\end{abstract}

Keywords: Cognitive Radio, Data Rate, Transmitter Power, Adhoc Networks.

\section{INTRODUCTION}

CR network, used first time in 1998 by Joseph Mitola, is an intelligent wireless communication system which senses the spectrum parameters and make changes accordingly to use the spectrum efficiently [1]. CR allows us to allocate the available spectrum dynamically to the unlicensed users whenever the licensed band is not used by the primary users for the transmission. It allows us to re-adjust various parameters at different layers of protocol stack so that various spectrum access methodologies can be supported, and the quality of service can be maintained for terminals [4].

\section{Architecture:}

The cognitive radio architecture has components divided in 2 groups, where one group is the primary network while the other is the CR network [2].

(1) Primary network:

Primary network is the network that has the license to transmit in the given frequency range. It consists of two parts:

(a) Primary User: A primary user is the user which has the right to access the given spectrum. The primary base station maintains the network access of a primary user which should not get altered by the operations of a secondary user during that time.

(b) Primary Base Station: The primary base station is like the base station in mobile radio networks. It supervises the use of spectrum among the primary users. It does not have the ability to extend the spectrum access to the secondary user.

\section{(2) Cognitive Radio network:}

Cognitive radio network is different from the primary network. It is an unlicensed network. This network can only access the spectrum band when there are spectrum holes [5]. A cognitive radio consists of:

(a) Cognitive User: It employs additional methods like spectrum holes identification, spectrum sensing etc. so that it can use the spectrum holes and satisfies its service needs without affecting the primary users.

(b) CR Base Station: Major function of CR base station is to detect the free bands and providing the access to
Cognitive Users without affecting performance of primary users.

(c) Spectrum Broker: This is the main part which allows various CR Networks to use the available spectrum resources. Spectrum information can be managed by the help of the spectrum broker which allows many CR Networks to co-exist.

Cognitive Radio Network can be categorized based on the infrastructure support. First one is centralized network, which is infrastructure based while the other one is CRAHN (Cognitive Radio Ad Hoc Network), which is infrastructureless network.

The centralized CR Network has a central node or an access point in case of wireless LANs. The central network node may be a base station. Point to point connections can be used to access the base station. CUs communicates with each other using the base station. If they belong to different base stations, then the routing takes place using the core network.

CRAHNs are different from the centralized networks as they don't have infrastructure for communication. A cognitive radio user can directly transfer the data to other CUs falling within its range of communication. It establishes point to point communication links for this process. They can use the freely available spectrum band, e.g. $2.4 \mathrm{GHz}$ band. Or they can make use of spectrum holes when available in the licensed bands [3]. Functions:

1. Spectrum Sensing: It is the core function of cognitive radio network which identify the holes in the spectrum bands of primary network and therefore increase the efficiency of the spectrum.

2. Spectrum Management: The secondary users then find the best spectrum band for communication.

3. Spectrum Mobility: When secondary user moves from a spectrum band to other spectrum band, it is known as spectrum mobility. It is done when the primary user want to use the band which secondary user is using.

4. Spectrum Sharing: To prevent the interference among the primary and the secondary users, this technique comprises power and channel assignments.

Challenges: 
Software-Defined Radio is a part that can't be ignored while talking about cognitive radio. These include:

(a) Attaining enough amount of computational capacity so that wideband spectra including large bit-rates can be processed within affordable costs.

(b) Security concerns that arises due to its flexibility.

(c) Planning an analogue radio system and providing translation between digital and analogue domain, mainly in high rate transmissions.

(d) Writing various application programs for different applications.

There are other challenges we face in CR Networks. We try to utilize network performance efficiently for a multi-node network. As we know, actions related to lower layers usually affect the performance of a network. E.g. the data rates between two nodes can be determined by computing the capacity of the link in case of a scheduling. We must make sure that the link is in operation in routing in upper layer before we make the link active for scheduling. We should not keep those links in mind for scheduling which are not used for routing. This shows that the layers are dependent on each other, and thus, a good cognitive radio network performance can be obtained by employing a cross layer design. The crosslayer design is often composite and complicated. The design space gets expended when we talk about its mathematical significance, involving many sets of integer variables, and in return, making it more complex. Also, linearity may not be maintained which adds to the difficulties and challenges faced by a cognitive radio system. These challenges make it difficult to maintain the optimum performance and efficiency. And the spectrum is thus, not fully utilized. These limitations push us to look for better concepts and innovations in algorithm plan.

\section{SURVEY OF RELEVANT LITERATURE}

In [6], an overview of cognitive radio system sensing techniques such as local spectrum sensing technique based on hypothesis testing criteria etc. It also include various techniques to improve the sensing performance. Further it also include various spectrum sharing technique.

In [7], spectrum sensing technique that is blind source separation method based on kurtosis metrics is used in which spectrum is sensed when the unauthorized user uses that spectrum.

$\mathrm{n}$ [8], review of implementation of unique features of cognitive radio technology such as spectrum location and environmental awareness. Also discuss the dynamic nature of cognitive radio system along with limitations, issues and mitigation techniques.

In [9], cognitive ad-hoc network using reinforcement learning (RL) schemes for protocol design is discussed. Also provide model to apply RL techniques and benefits of using these techniques in dynamic conditions of spectrum allocation.

In [10], discussed some challenges in CRAN which are not in traditional wireless ad-hoc network and their impact on the algorithm designing for the basic problem in cognitive radio ad-hoc network. It mainly considered two problems that are capacity maximization and connectivity problem and reformulated them.

In [11], optimization of capacity of cognitive radio spectrum depend on the number of secondary user and power of secondary user which varies with channel characteristic and usage of spectrum band by primary user. By using joint average interference power and peak transmit power , maximum capability of secondary user is obtained on fading channels and also in multiple quadrature amplitude modulation. Also results and comparison of spectrum sharing system's performance in different working environments are given.

In [12], overview of cognitive radio technology that solves the problem of spectrum scarcity and functions of three layers: physical (PHY) layer, medium access control (MAC) layer and network layer. Function of physical layer includes the techniques to sense the spectrum, MAC layer includes the sensing scheduling schemes and MAC protocols., network layer includes routing and quality control.

In [13], fast algorithm is used to solve power allocation problem for orthogonal frequency division multiplexing (OFDM). In this algorithm, newton step with $\mathrm{O}\left(\mathrm{N}^{3}\right)$ complexity in barrier method is placed with approximate linear complexity based on structure of optimization problem.

In [14], reckoned a framework where low power devices for secondary users are used to form cognitive radio network along with the primary and secondary users operating concurrently. It proposed power control problem for cognitive radio network to maximize efficiency of energy of cognitive users ensuring the quality of both users. Also suggested the combined power and admission control procedure. Considering co-channel interference from heterogeneous system along with ensurity of priority of primary user.

In [15], as the number of active primary user increases, the chances of blocking of secondary also increases to maintain the interference at primary user (PU) receiver and quality of service (QoS) of secondary users that is secondary users have to adjust their transmission power every time to maintain the interference and quality of service. For this problem, a flow maintenance and power control method is designed which is known as flow based power control (FBPC). This method includes two stage : first stage finds the maximum size of best set of flow and second stage is to optimize the minimal flow capacity using power control. To lessen its complexity in first stage, flow combination metric is designed to determine the best combination of flow.

In [16], focuses on primary user influence because previous work mainly focuses on secondary user interaction. This paper developed a net power gain control model in which primary users share their spectrum with secondary users for which they are recompensed. This model provide the energy efficient transmission for secondary users, ensuring the quality requirement of primary users and providing the rewards to primary user.

In [17], focuses on secondary user whose function depend on its peak transmit power constraint and an average interference constraint at primary receiver. In this paper we discussed about the power adaption techniques for optimizing the secondary users SNR and capacity. This technique degrades the secondary transmit power because of increase of primary user.

In [18], a model for maximizing the performance of secondary link is designed concerning average spectrum efficiency, considering the secondary user's quantal channel state information (CSI). In this paper we designed a algorithm for calculating the quantized channel state information along with discrete power utilized at cognitive transmitter for each quantal CSI level in order for the maximum utilization of 
spectrum. Performance results provide the bits necessary to show CSI to attain the maximal average spectrum efficiency.

In [19], firstly distance of SU (secondary user) from the PU (primary user) is calculated so that SU can utilize the channel without spectrum sensing. If spectrum sensing is required then we calculate the sense parameters and transmission power for optimizing the throughput of secondary user otherwise secondary user detects the PU and forbids the primary user receiver from decrypting its data. Therefore we can say that outage of PU depends on probability of detection. This outage probability says that if transmitter power of SU is very low or very high then only spectrum sensing is required otherwise not.

In [20], has taken into account the spectrum sensing scheduling problem in cognitive radio networks. In their work each secondary user (SU) is free to determine whether it want to take part in cooperative spectrum sensing or not. If it doesn't want to participate, SU becomes an eavesdropper. It will help in conserving the energy for spectrum sensing but will affect the spectrum sensing performance. For this they considered two questions : first one " whether to participate or not in spectrum sensing?" and second one "which channel is to sense?”. For the first question each secondary user decides on the basis of utility history. If higher utility comes out then each SU takes action more frequently. For the second question they develop an entropy based coalition formation algorithm in which every SU that select the same channel for sensing form an alliance.

In [21], this paper give a detailed overview of first worldwide wireless standard based on the cognitive radio: IEEE 802.22. It consist of cognitive radio based wireless regional area network (CR-WRAN) physical (PHY) layer and medium access control layer (MAC layer) . 802.22 requires reuse fallow TV spectrum without affecting TV receivers. It mainly consist of some advanced techniques that provides dynamic spectrum management.

In [22], discussed various spectrum management techniques in cognitive radio networks. It proposed the pre-emptive resume priority (PRP) M/G/1 queuing model for performance evaluation of these spectral management methods. Using this model, they derived a new measure of performance i.e. overall system time. This new measure is the time period when data comes at the system till the finishing of the complete transmission. High priority primary users provide numerous interruptions that will affect the low priority secondary users. All the spectral management techniques such as spectrum sensing algorithm, spectrum handoff algorithm are enquired to decrease overall system time.

In [23], provides scanning free-region where cognitive radio system can co-occur with a primary network. By using these two network together system throughput increases and also it provides the optimum utilization of radio spectrum. This paper proposed a model that helps us to calculate a region where the location uncertainty value is known. By using this model a maximum tolerable value of location uncertainty is calculated so that there is accurate performance of concurrent transmission scheme.

In [24], suggested a dynamic programming based algorithm which will increase average rate for cognitive radio (CR) link considering total energy budget and cognitive radio-to-primary radio disturbance. The suggested method models the primary radio as two-state Markov chain. This model derived the optimum power control and rate control strategy for each time slot. Results of the suggested algorithm shows a good performance improvement in average rate along with keeping CR-to-PR disturbance below a given level.

In [25], suggested a generic model for power control and a methodical design for optimum power sharing in cognitive radio networks. This paper consider the constraint in which nodes in the cognitive radio networks switch to different band so that secondary users(SU) transmission remain lucid to the primary users (PU).

In [26], problem of interference is discussed which occurs when cognitive radio (CR) uses a same band as that of the primary user (PU) without noticing the presence of PU. This interference problem leads to the degradation of the quality of service of the primary receivers. For this problem an adaptive power control scheme is used which calculates the approximate distance between the PU and CU by considering the SNR as proxy. With the help of this information, interference is avoided as CR continuously adjust its maximal transmit power. Finally the mathematical results shows the validation of this algorithm.

\section{CONCLUSION}

This paper described the cognitive radio networks which increased the utilization of the spectrum bands as compared to the conventional wireless networks. Various algorithms and techniques proposed in the field of CR networks which increased its efficiency and power by various researchers are also discussed.

\section{REFERENCES}

[1] J.Mitola and G.Q.Maguire, "Cognitive radio: making software radios more personal”, IEEE Personal Commun., vol.6, no.4, pp.13-18, Aug. 1999.

[2] Ian F. Akyildiz, Won-Yeol Lee, Kaushik R. Chowdhury, "CRAHNs: Cognitive radio ad hoc networks", Article on press, Journal (Elsevier), 7(5):810-836, Jul. 2009.

[3] Md. Arafatur Rahman, "Cognitive Radio Ad-hoc Networks: A Routing Perspective. PhD Dissertation”, University of Naples, Federico II, Apr. 2013.

[4] Prabhjot Kaur, Moin Uddin, Arun Khosla "Adaptive Bandwidth Allocation Scheme for Cognitive Radios", doi: 10.4156/ijact.vol2.issue2.3, June 2010

[5] Cunhao Gao, "Some Modeling and Optimization Problems in Cognitive Radio Ad Hoc Networks", Master's Dissertation, Virginia Polytechnic Institute and State University, Blacksburg Virginia, September 2009

[6] Lu et al., "Ten years of research in spectrum sensing and sharing in cognitive radio,” EURASIP Journal on Wireless Communications and Networking, 2012.

[7] S.S. Ivrigh, S.Mohammad-Sajad Sadough and S. Ali Ghorashi, "A Blind Source Separation Technique for Spectrum Sensing in Cognitive Radio Networks Based on Kurtosis Metric," International eConference on Computer and Knowledge Engineering (ICCKE), pp. 333-337, 2011.

[8] H Celebi, Ismail Guvenc, S Gezici and H Arslan, "CognitiveRadio Systems for Spectrum, Location and Environmental Awareness”, IEEE Antennas and Propagation Magazine, vol. 52, pp. 41-61, 2010.

[9] M. Di Felice, K.R Chowdhury, Chang Wu, L. Bononi and Waleed Meleis, "Learning-Based Spectrum Selection in Cognitive Radio Ad Hoc Networks”, E. Osipov et al. (Eds.): WWIC 2010, LNCS 6074, pp. 133-145, 2010.

[10] Q-S Hua, H. Tan, Y. Wang, H. Li, D. Yu, Francis C.M. Lau and C. Wu, "Computing Capacity and Connectivity in Cognitive Radio Ad-Hoc Networks”, International 
Symposium on Pervasive Systems, Algorithms and Networks, pp. 9-16, 2012.

[11] V. Asghari and S. Aissa, "Adaptive Rate and Power Transmission in Spectrum-Sharing Systems," IEEE Trans. Wireless Communication, vol. 9, no. 10, pp. 3272-3280, 2010.

[12] Y-C Liang, K-C Chen, G. Ye Li and P. Mahonen, "Cognitive Radio Networking and Communications: An Overview", IEEE Transactions on Vehicular Technology, vol. 60, no. 7, pp.3386-3407, 2011.

[13] S. Wang, F. Huang and Z-H Zhou, "Fast Power Allocation Algorithm for Cognitive Radio Networks", IEEE Communication Letters, vol. 15, no. 8, pp. 845-847, 2011.

[14] L. Qian, X. Li, J. Attia, Z. Gajic, "Power Control for Cognitive Radio Ad Hoc Networks", IEEE LANMAN Workshop, 2007.

[15] Ba Lam To, Thi Mai Trang Nguyen and Guy Pujolle, "Flow-Based Control in Cognitive Radio Ad-hoc Network", International Wireless Communications and Mobile Computing, pp. 130-135, 2012.

[16] T. Li and Sudharman K. Jayaweera, "A Novel PrimarySecondary User Power Control Game for Cognitive Radios”, International Symposium on Informatio Theory and its Applications, pp. 1-6, 2008.

[17] S. Srinivasa and S. A. Jafar, "Soft Sensing and Optimal Power Control for Cognitive Radio," IEEE Trans. Wireless Communication, vol. 9, no. 12, pp. 3638-3649, 2010.

[18] Mohamed M.Abdallah, Ahmed H.Salem, Mohamed-Slim Alouini, Khalid A.Qaraqe, “ Adaptive Discrete Rate and Power Transmission for Spectrum Sharing Systems”, IEEE Transactions on Wireless Communications, vol. 11, no. 4, pp. 1283-1289, 2012.
[19] E.C.Y. Peh, Y-C Liang and Y.Zeng, "Sensing and power control in cognitive radio with location information," ICCS, 2012, PP. 255-259.

[20] Hongjuan Li, Xiuzhen Cheng, Keqiu Li, Xiaoshuang Xing and Tao Jing, "Utility-Based Cooperative Spectrum Sensing Scheduling in Cognitive Radio Networks", in Proceeding IEEE INFOCOM, pp. 165-169, 2013.

[21] C. Cordeiro, K. Challapali, D. Birru, N. S. Shankar, "IEEE 802.22: the first worldwide wireless standard based on cognitive radios", First IEEE International Symposium New Frontiers in Dynamic Spectrum Access Networks DySPAN '05', pp. 328-337, 2005.

[22] L.-C. Wang, C.-W. Wang, "Spectrum management techniques with QoS provisioning in cognitive radio networks", IEEE International Symposium on Wireless Pervasive Computing, pp. 116-121, 2010.

[23] S. Montejo S, V. Alfonso Reguera, E. M. Garcia Fernandez, W. Godoy Junior, "Impact of location accuracy on concurrent transmission for cognitive ad hoc networks", IEEE Latin-American Conference on Communications, pp. 1-6, 2011.

[24] Long Gao, Peng Wu and Shuguang Cui, "Power and Rate Control with Dynamic Programming for Cognitive Radios”, IEEE GLOBECOM 2007- IEEE Global Telecommunications Conference, pp. 1699-1703, 2007.

[25] Ba-Lam To, Hicham Khalife and Naceur Malouch, “A General Framework for Assisted Power Control in Cognitive Radios Networks", IEEE International Symposium on Personal, Indoor and Mobile Radio Communications, pp. 1648-1653, 2010.

[26] M. Elalem, L. Zhao and Z. Liao, "Interference mitigation using power control in cognitive radio networks," IEEE Vehicular Technology Conference, pp. 1-5, 2010. 